\section{Elektrodialytische Regenerierung von Ionenaustauscher-Harzen}

\author{
Jürgen Johann und Gerhart Eigenberger*
}

Ionenaustauscher-Harze werden überwiegend zur Abtrennung unerwünschter Ionen aus wäßrigen Lösungen eingesetzt. Die Arbeitskapazität der Harze ist jedoch beschränkt. Deswegen müssen die Harze von Zeit zu Zeit regeneriert werden. Die Regenerierung wird meist im Gegenstrom, bei der Wasservollentsalzung mit Säure und Lauge, bei der Enthärtung mit einer $\mathrm{NaCl}-\mathrm{Lösung}$, durchgeführt. Nachteilig ist dabei vor allem der überstöchiometrische Bedarf an Regenerierchemikalien, insbesondere bei stark sauren Kationenaustauscher-Harzen. Des weiteren ist im Falle schwermetallionenhaltiger Regenerate eine zusätzliche Aufbereitung derselben z. B. durch Fällung notwendig.

Ein neueres Verfahren ist die elektrodialytische Regenerierung der Harze $[1,2]$. Orientierende Untersuchungen hierüber gibt es von Strathmann [3]. Dieses Prinzip wurde zu einem kontinuierlichen Verfahren weiterentwickelt $[4,9]$. Im Unterschied zu [5] wird nur eine Ionenaustauscher-Sorte zwischen den Membranen eingesetzt. Die nachstehend beschriebenen experimentellen Ergebnisse am Beispiel der Cu-Ionenabtrennung zeigen die Vorteile im Vergleich zu den herkömmlichen Regenerierverfahren.

\section{Versuchsapparatur}

Abb. 1 zeigt den Aufbau des Moduls im Falle eines Kationenaustauscher-Harzes. Das Harz befindet sich in einer Kammer $I$, welche durch zwei Kationenaustauscher-Membranen $K$ begrenzt wird. Vor bzw. hinter dieser Kammer $I$ befindet sich, durch eine Anionenaustauscher-Membran $A$ voneinander getrennt, je eine Säure- und Konzentratkammer $S, C$. An den beiden Enden des Moduls sind die Elektrodenkammern $E$ mit Anode und Kathode angeordnet.Während des Betriebs wird eine elektrische Spannung an die Elektroden angelegt. Das Feed durchströmt die Ionenaustauscher-Kammern 1 , wobei der Austausch der unerwünschten Ionen wie in einem konventionellen Ionenaustauscher-Bett erfolgt. Unter der treibenden Kraft des elektrischen Feldes wandern aber gleichzeitig die $\mathrm{H}^{+}$-Ionen aus den Säurekammern $S$ über die Kationenaustauscher-Membranen in die Ionenaustauscher-Kammern $I$ und ersetzen die auf dem IonenaustauscherHarz befindlichen Kationen. Diese Ionen wandern über die nächste Kationenaustauscher-Membran in die darauffolgende Konzentratkammer $C$. Durch Anordnung einer Anionenaustau-

* Prof. Dr.-Ing. G. Eigenberger und Dr.-Ing. J. Johann, Institut für Chemische Verfahrenstechnik, Böblinger Straße 72, 7000 Stuttgart 1.

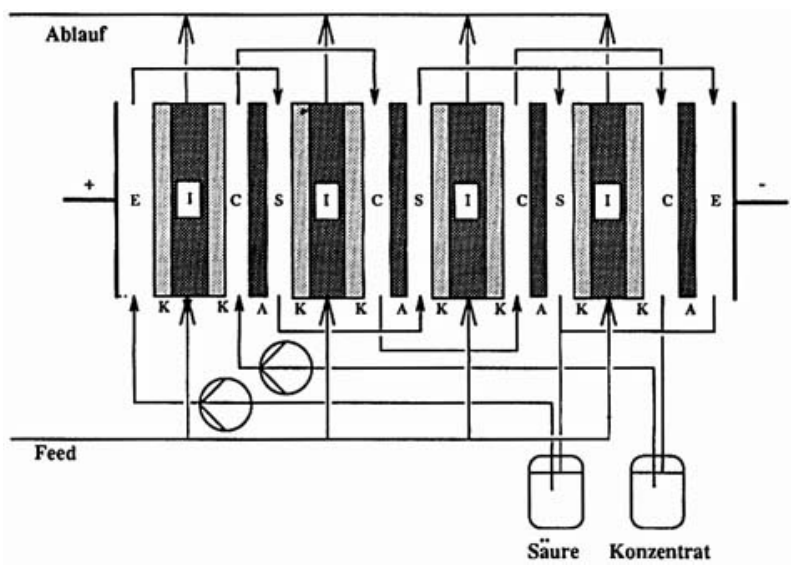

Abb. 1. Anlagenschema.

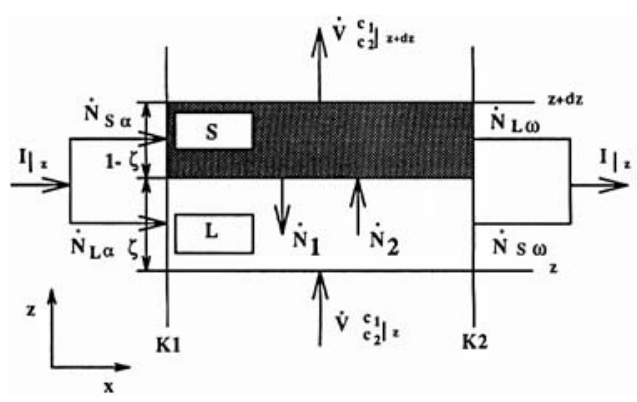

Abb. 2. Bilanzraum.

scher-Membran $A$ zwischen der Konzentratkammer $C$ und der nachfolgenden Säurekammer $S$ wird verhindert, daß die ausgetauschten Kationen in die Säurekammer $S$ eindringen.

\section{Modellbildung}

Wichtige Erkenntnisse über dieses Verfahren liefert die Kombination von gezielten Experimenten mit detaillierter Modellbildung und Rechnersimulation. Die Modellgleichungen werden an einem Volumenelement $\mathrm{d} V$ der Höhe $\mathrm{d} z$ aufgestellt (Abb. 2). Die Diskretisierung findet hier über der Verfahrenslänge ( $z$-Richtung) statt. Senkrecht dazu ( $x$-Richtung) wird die IonenaustauscherPackung als ideal durchmischt betrachtet. Für das Stoffsystem $\mathrm{CuSO}_{4}$ (Feed) und $\mathrm{H}_{2} \mathrm{SO}_{4}$ (Säurekreis) werden im folgenden die drei Ionensorten $\mathrm{Cu}^{2+}$ (Index $\left.i=1\right), \mathrm{H}^{+}($Index $i=2)$ und $\mathrm{SO}_{4}^{2-}$ (Index $i=3$ ) betrachtet. Aus der Stoffbilanzierung erhält man die folgenden Gleichungen für jede Ionensorte:1)

\section{Liquid-Phase:}

$$
\begin{aligned}
\varepsilon \mathrm{d} V \frac{\partial c_{\mathrm{i}}}{\partial t}= & \dot{V}\left(c_{\mathrm{i},\left.\right|_{\mathrm{z}}}-c_{\mathrm{i},\left.\right|_{\mathrm{z}+\mathrm{d} z}}\right)-D_{\mathrm{i}} A\left(\left.\frac{\partial c_{\mathrm{i}}}{\partial z}\right|_{z}-\left.\frac{\partial c_{\mathrm{i}}}{\partial z}\right|_{\mathrm{z}+\mathrm{d} z}\right)+ \\
& +\left(\dot{N}_{\mathrm{i}, \alpha}^{\mathrm{L}}-\dot{N}_{\mathrm{i}, \omega}^{\mathrm{L}}\right) \mathrm{d} A^{\mathrm{M}}-\frac{D_{\mathrm{ID}} \mathrm{d} A^{\mathrm{IAT}}}{\delta}\left(c_{\mathrm{i}}-c_{\mathrm{i}}^{\mathrm{GG}}\right)
\end{aligned}
$$

Dabei beschreiben die ersten drei Terme Speicherung sowie Konvektion und Dispersion der Ionensorte i längs der Strömungs-

1) Eine Zusammenstellung der Formelzeichen befindet sich am Schluß des Beitrags. 
koordinate $z$, der vorletzte Term den Austauschstrom $\dot{N}_{i}^{L}$ in die Flüssigphase über die linke und rechte Ionenaustauscher-Membran und der letzte Term den Austausch zwischen der Flüssigphase und der lonenaustauscher-Schüttung. Triebkraft dafür ist die Konzentrationsdifferenz zur Gleichgewichtskonzentration $\left(c_{\mathrm{i}}^{\mathrm{GG}}\right)$. Als Transportwiderstand wurde ein (äußerer) Diffusionsfilm der Dicke $\delta$ postuliert; $D_{\mathrm{ID}}$ kennzeichnet den Interdiffusionskoeffizienten des Stoffaustauschs.

\section{Solid-Phase:}

$$
\begin{aligned}
(1-\varepsilon) \mathrm{d} V \frac{\partial \bar{c}_{\mathrm{i}}}{\partial t}= & \frac{D_{1 \mathrm{D}} \mathrm{d} A^{\mathrm{IAT}}}{\delta}\left(c_{\mathrm{i}}-c_{\mathrm{i}}^{\mathrm{GG}}\right)+ \\
& +\left(\dot{N}_{\mathrm{i}, \alpha}^{\mathrm{s}}-\dot{N}_{\mathrm{i}, \omega}^{\mathrm{S}}\right) \mathrm{d} A^{\mathrm{M}} .
\end{aligned}
$$

Für die ortsfeste Ionenaustauscher-Phase mit der Ionenkonzentration $\bar{c}_{\mathrm{i}}$ wird ebenfalls ein direkter Austauschstrom $\dot{N}_{\mathrm{i}}^{\mathrm{S}}$ von den bzw. in die seitlich angrenzenden Ionenaustauscher-Membranen angesetzt.

Gemäß Abb. 2 wird der aufgeprägte Elektrodenstrom $I$ zum einen in Form der Ionenströme $\dot{N}_{i}^{\mathrm{s}}$ durch die IonenaustauscherSchüttung, zum anderen in Form der Ionenströme $\dot{N}_{i}^{\mathrm{L}}$ durch die die Ionenaustauscher-Körner umgebenden Flüssigkeit transportiert. Maßgebend für die Aufteilung des Stromes $I$ sind die Ohmschen Widerstände in der Parallelschaltung von Solid- und Liquid-Phase, die wiederum von den Ionenkonzentrationen und den Ionenbeweglichkeiten in den jeweiligen Phasen abhängen.

Zur Ableitung des Zusammenhangs ist es zweckmäBig, die Abhängigkeit der Ionenströme $\dot{N}_{i}$ jeder Phase von der elektrischen Stromdichte $i(z)$ zunächst mit der formal definierten Transportzahl $t_{i}$ zu beschreiben:

L-Phase: $\dot{N}_{\mathrm{i}}^{\mathrm{L}}=\frac{t_{\mathrm{i} . \mathrm{L}} i(z)}{z_{\mathrm{i}} F}$,

S-Phase: $\dot{N}_{\mathrm{i}}^{\mathrm{s}}=\frac{t_{\mathrm{i},} \mathrm{s}^{i}(z)}{z_{\mathrm{i}} F}$,

wobei für die Transportzahl gilt [1]:

$$
\begin{aligned}
& \text { L-Phase: } t_{\mathrm{i}, \mathrm{L}}=\frac{z_{\mathrm{i}}^{2} u_{\mathrm{i}} c_{\mathrm{i}}}{\sum_{i=1}^{3}\left(z_{\mathrm{i}}^{2} u_{\mathrm{i}} c_{\mathrm{i}}\right)+\sum_{i=1}^{2}\left(z_{\mathrm{i}}^{2} \bar{u}_{\mathrm{i}} \bar{c}_{\mathrm{i}}\right)+\bar{u}_{0} X}, \\
& \text { S-Phase: } t_{\mathrm{i}, \mathrm{S}}=\frac{z_{\mathrm{i}} \bar{c}_{\mathrm{i}}\left(z_{\mathrm{i}} \bar{u}_{\mathrm{i}}+\bar{u}_{0}\right)}{\sum_{i=1}^{3}\left(z_{\mathrm{i}}^{2} u_{\mathrm{i}} c_{\mathrm{i}}\right)+\sum_{i=1}^{2}\left(z_{\mathrm{i}}^{2} \bar{u}_{\mathrm{i}} \bar{c}_{\mathrm{i}}\right)+\bar{u}_{0} X} \text {. }
\end{aligned}
$$

Darin sind $u_{\mathrm{i}}, \bar{u}_{\mathrm{i}}$ die Ionenbeweglichkeiten in Liquid- und SolidPhase und $X=\sum_{i=1}^{n} z_{i} \overline{\mathrm{c}}_{\mathrm{i}}$ die Festionenkonzentration des Ionenaustauschers. Dabei wird vorausgesetzt, daß nur Gegenionen $\left(\mathrm{Cu}^{2+}\right.$ und $\mathrm{H}^{+}$) den Stromtransport im Ionenaustauscher übernehmen.

Für den Gesamtstrom 1 gilt:

$$
I=b \int_{0}^{l} i(z) \mathrm{d} z
$$

mit der Kammerbreite $b$ bzw. der Membranfläche $\mathrm{d} A=b \mathrm{~d} z$.

Die Stromdichte $i(z)$ berechnet sich nach dem Ohmschen Gesetz aus der Leitfähigkeit $\varkappa_{\mathrm{p}, \mathrm{z}}$ des Abschnitts $\mathrm{d} z$ (IonenaustauscherHarz plus Liquid-Phase) multipliziert mit dem Potentialgradienten a $p / \partial x$ senkrecht zur Strömungsrichtung: $i(z)=-\varkappa_{p, z} \frac{\partial \varphi}{\partial x}$

Daraus folgt:

$l=-b \int_{0}^{l} x_{\mathrm{p}, \mathrm{z}} \frac{\partial \varphi}{\partial x} \mathrm{~d} z$

Mit der Annahme, daß der Potentialabfall $\partial \varphi / \partial x$ in der Kammer über der Verfahrenslänge konstant bleibt, erhält man aus den Gln. (8) und (9) die Gleichung zur Berechnung der Stromdichte $i(z)$ bei vorgegebenem Gesamtstrom I:

$$
i(z)=x_{\mathrm{p}, z} \frac{I}{b \int_{0}^{1} x_{\mathrm{p}, \mathrm{z}} \mathrm{d} z} .
$$

Die Leitfähigkeit der Ionenaustauscher-Packung $x_{p, z}$ setzt sich additiv aus den Leitfähigkeiten von L-Phase (Flächenanteil $\zeta$ ) und S-Phase (Flächenanteil $(1-\zeta))$ zusammen:

$x_{\mathrm{p}, \mathrm{i}}=\zeta \varkappa_{\mathrm{L}, \mathrm{i}}+(1-\zeta) x_{\mathrm{S}, \mathrm{i}}$

Die Leitfähigkeiten von Liquid- und Solid-Phase lassen sich wiederum aus den Ionenbeweglichkeiten und den Ionenkonzentrationen bestimmen:

L-Phase: $\varkappa_{\mathrm{L}, \mathrm{i}}=F \sum_{i=1}^{3} z_{\mathrm{i}}^{2} u_{i} c_{\mathrm{i}} \quad$,

S-Phase: $\varkappa_{\mathrm{S}, \mathrm{i}}=F \sum_{i=1}^{3}\left(z_{\mathrm{i}}^{2} \bar{u}_{\mathrm{i}} \bar{c}_{\mathrm{i}}+\bar{u}_{i} X\right)$

Für den Interdiffusionskoeffizienten $D_{\mathrm{ID}}$ folgt nach [6]:

$D_{\mathrm{ID}}=D_{1} D_{2} \frac{z_{1}^{2} c_{1}+z_{2}^{2} c_{2}+z_{3}^{2} c_{3}}{D_{1} z_{1}^{2} c_{1}+D_{2}\left(z_{2}^{2} c_{2}+z_{3}^{2} c_{3}\right)} \quad$,

und für die Grenzschichtdicke $\delta$ gilt nach [8]:

$\delta=0,1 r \operatorname{Re}^{-0,84}$.

Die Gleichgewichtskonzentrationen $c_{\mathrm{i}}^{\mathrm{GO}}$ an der äußeren Oberfläche der Ionenaustauscher-Körner können aus der formalen Anwendung des Massenwirkungsgesetzes beim IonenaustauschProzeB nach [7] berechnet werden.

Aus den Gln. (4) bis (15) erhält man auf diese Weise für den Konzentrationsverlauf von $\mathrm{Cu}^{2+}$ (Index $i=1$ ) in L-Phase und S-Phase die nachstehenden Modellgleichungen:

\section{L-Phase:}

$$
\begin{aligned}
& \frac{\partial c_{1}}{\partial t}+\frac{v}{\varepsilon} \frac{\partial c_{1}}{\partial z}-\frac{D_{1}}{\varepsilon} \frac{\partial^{2} c_{1}}{\partial z^{2}}= \\
& \left(t_{1 . \mathrm{L}}^{\alpha}-t_{1 . \mathrm{L}}^{\mathrm{ta}}\right) \varkappa_{\mathrm{p}, \mathrm{z}} \frac{I}{\left(b \int_{0}^{l} \varkappa_{\mathrm{p}, z} \mathrm{~d} z\right) z_{1} F \mathrm{~d} \varepsilon}-\frac{D_{\mathrm{ID}} a_{v}}{\delta \varepsilon}\left(c_{1}-c^{1, \mathrm{GG}}\right) .
\end{aligned}
$$

Die Randbedingungen lauten im Zulauf: $c_{1}(t, z=0)=c_{1, z u}$, im Auslauf: $\left.\frac{\partial c_{1}}{\partial z}\right|_{z=1}=0$.

Als Anfangsbedingung gilt: $c_{1}(t=0, z)=c_{1,0}$. 
S-Phase:

$$
\begin{aligned}
\frac{\partial \bar{c}_{1}}{\partial t}= & \frac{D_{\mathrm{ID}} a_{v}}{\delta(1-\varepsilon)}\left(c_{1}-c^{1, \mathrm{GG}}\right)+ \\
& +\left(t_{1, \mathrm{~s}}^{\alpha}-t_{1, \mathrm{~S}}^{\omega}\right) \varkappa_{\mathrm{p}, \mathrm{z}} \frac{I}{\left(b \int_{0}^{l} \varkappa_{\mathrm{p}, \mathrm{z}} \mathrm{d} z\right) z_{1} F d(1-\varepsilon)},
\end{aligned}
$$

Anfangsbedingung: $\bar{c}_{1}(t=0, z)=\bar{c}_{1,0}$. Desweiteren wird angenommen, daß kein $\mathrm{Cu}^{2+}$-Transport über die KationenaustauscherMembran $K 1$ (Abb. 2) stattfindet. Hieraus folgt: $t_{1, \mathrm{~L}}^{\alpha}=t_{1, \mathrm{~S}}^{\alpha}=0$.

\section{Ergebnisse}

In Ionenaustauscher-Schüttungen ohne elektrisches Feld wandert die Konzentrationsfront mit einer gleichmäßigen Frontwanderungsgeschwindigkeit durch das gesamte Bett, Abb. 3. Sobald die Ablaufkonzentration einen bestimmten Grenzwert überschreitet, muß die Schüttung regeneriert werden. Ein stationärer Zustand stellt sich nicht ein.

Im Unterschied hierzu wird bei Einschalten eines Elektrodenstromes die Frontwanderungsgeschwindigkeit $v^{\mathrm{F}}$ mit fortschreitender Versuchsdauer kleiner, Abb. 4. Die Front wandert nicht mehr aus der Schüttung heraus, sondern stabilisiert sich. Die übertragene Cu-Menge ist dabei abhängig von der eingestellten Stromdichte.

Wie ein Vergleich von Abb. 3 und 4 erkennen läßt, ist die Betriebszeit des Ionenaustauschers bis zum Durchbruch bei einer mittleren Stromdichte von $10 \mathrm{~mA} / \mathrm{cm}^{2}$ um den Faktor 2 größer geworden.

Eine vollständige Überführung der Cu-Ionen gelingt bei höheren Stromdichten. Abb. 5 zeigt einen Vergleich zwischen Rechnung und Messung für einen Versuch bei einer mittleren Stromdichte von $i=16,7 \mathrm{~mA} / \mathrm{cm}^{2}$.

Unter diesen Bedingungen stellt sich nach längerer Betriebszeit ein stationärer Zustand ein, bei dem die Cu-Konzentration im

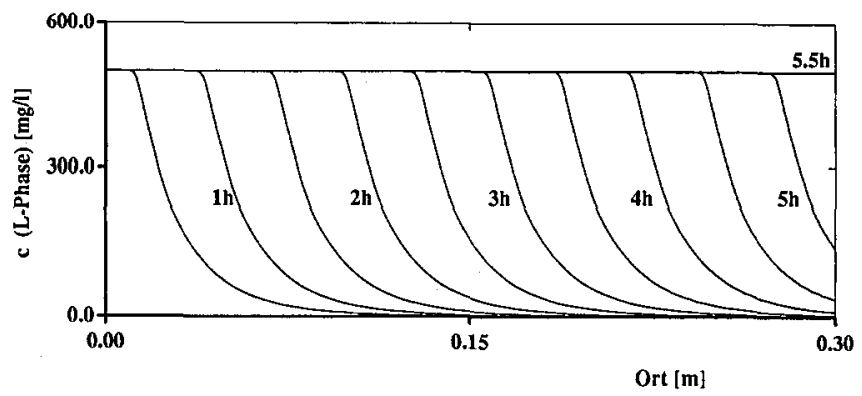

Abb. 3. Ortsprofile in einer lonenaustauscher-Schüttung ohne elektrisches Feld.

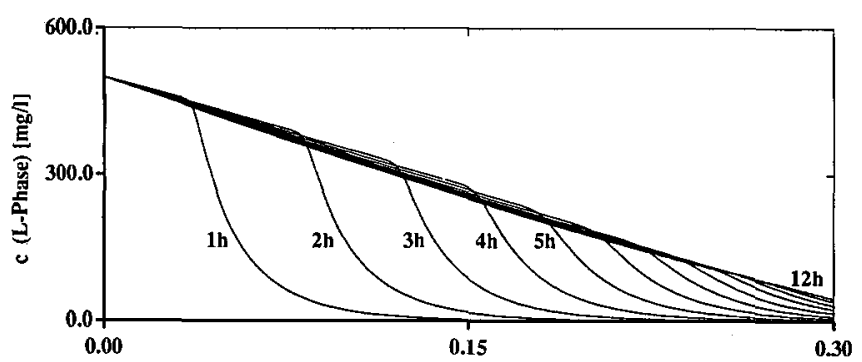

Ort [m]

Abb. 4. Ortsprofile der Cu-lonenkonzentration in der Flüssigphase, mittlere Stromdichte $i=10 \mathrm{~mA} / \mathrm{cm}^{2}$.

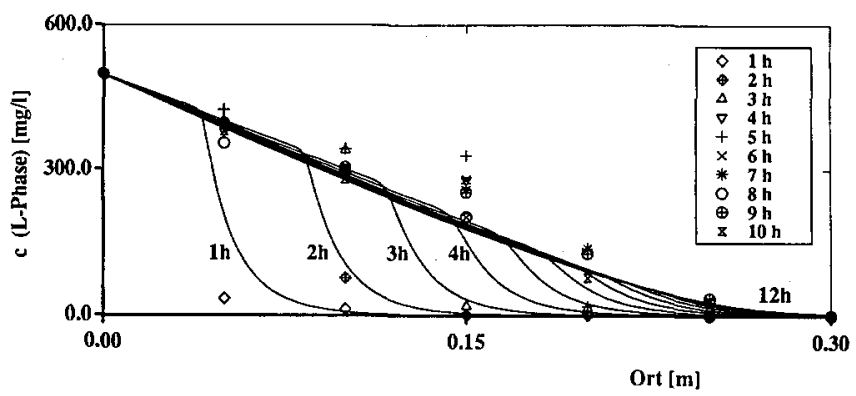

Abb. 5. Cu-Konzentrationsprofile (L-Phase) bei einer mittleren Stromdichte $i=16,7 \mathrm{~mA} / \mathrm{cm}^{2}$; Vergleich zwischen Rechnung (durchgezogene Linien) und Messung (Symbole).

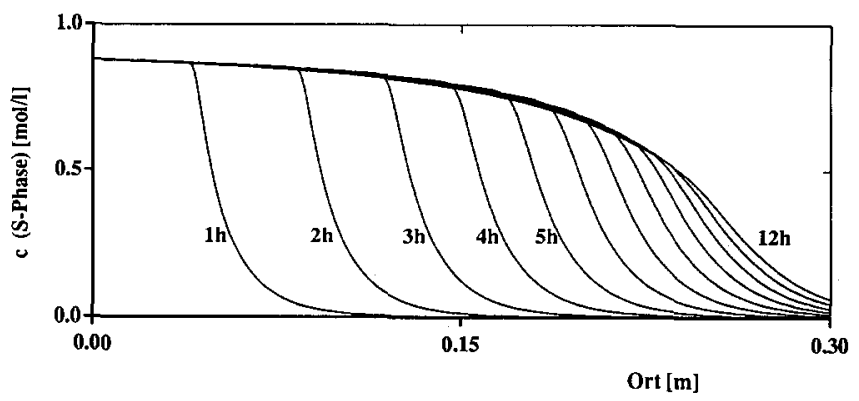

Abb. 6. Cu-Beladungsprofile in der lonenaustauscher-Schüttung.

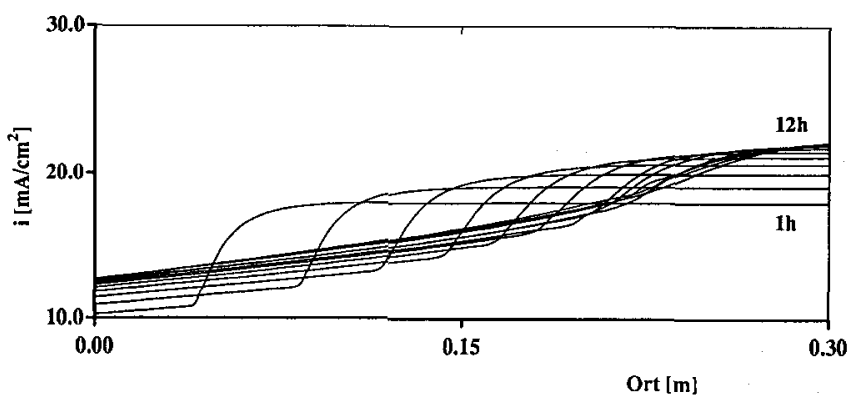

Abb. 7. Berechnete Stromprofile in der IonenaustauscherSchüttung.

Ablauf nahezu Null beträgt. Die gesamte Cu-Menge wird innerhalb der Schüttung übertragen. Abb. 6 zeigt die dazugehörigen gerechneten Beladungsprofile des Ionenaustauschers. Die $\mathrm{Cu}$ Beladung am Ende der Schüttung ist deutlich geringer als im vorderen Teil.

Aus den gerechneten Stromprofilen in Abb. 7 erkennt man, daß die Stromdichte am Ende der Schüttung stark ansteigt. Grund hierfür ist die hohe elektrische Leitfähigkeit des Harzes in der $\mathrm{H}^{+}$-Form.

Die hohe Stromdichte am Ende des Verfahrensweges bewirkt eine ständige Regenerierung des Harzes und verhindert somit einen Durchbruch der Cu-Front.

Eingegangen am 13. Januar 1992 [K 1399]

\section{Formelzeichen}

$\begin{array}{lll}A & {[\mathrm{~m}]} & \text { Membranfläche } \\ c & {\left[\mathrm{~mol} / \mathrm{m}^{3}\right]} & \text { Ionenkonzentration, L-Phase } \\ \vec{c} & {\left[\mathrm{~mol} / \mathrm{m}^{3}\right]} & \text { Ionenkonzentration, S-Phase }\end{array}$




$\begin{array}{lll}D & {\left[\mathrm{~m}^{2} / \mathrm{s}\right]} & \text { Diffusionskoeffizient } \\ F & {[\mathrm{~A} \mathrm{~s} / \mathrm{val}]} & \text { Faraday-Konstante } \\ i & {\left[\mathrm{~A} / \mathrm{m}^{2}\right]} & \text { Stromdichte } \\ \dot{N} & {\left[\mathrm{~mol} /\left(\mathrm{m}^{2} \mathrm{~s}\right)\right]} & \text { Molen- bzw. Ionenstrom } \\ r & {[\mathrm{~m}]} & \text { Kornradius } \\ \operatorname{Re} & {[-]} & \text { Reynolds-Zahl } \\ t & {[\mathrm{~s}]} & \text { Zeit } \\ t & {[-]} & \text { Transportzahl } \\ u & {\left[\mathrm{~m}^{2} /(\mathrm{V} \mathrm{s})\right]} & \text { Ionenbeweglichkeit, L-Phase } \\ \bar{u} & {\left[\mathrm{~m}^{2} /(\mathrm{V} \mathrm{s})\right]} & \text { Ionenbeweglichkeit, S-Phase } \\ v & {[\mathrm{~m} / \mathrm{s}]} & \text { Konvektionsgeschwindigkeit } \\ V & {\left[\mathrm{~m}^{3}\right]} & \text { Volumen } \\ V & {\left[\mathrm{~m}^{3} / \mathrm{s}\right]} & \text { Volumenstrom } \\ w & {[-]} & \text { Porenanteil im Ionenaustauscher } \\ x & {[-]} & \text { Koordinate senkrecht zur Strömungs- } \\ & & \text { richtung } \\ z & {[-]} & \text { Koordinate in Strömungsrichtung } \\ z & {[-]} & \text { Wertigkeit } \\ X & {\left[\mathrm{~mol} / \mathrm{m}^{3}\right]} & \text { Kapazität des Ionenaustauschers } \\ & & \left.\text { ( = } \sum_{i=1}^{n} z_{i} \bar{c}_{\mathrm{i}}\right) \\ & & \text { Grenzschichtdicke } \\ \delta & {[\mathrm{m}]} & \text { Leerraumanteil } \\ \varepsilon & {[-]} & \text { Potential } \\ \varphi & {[\mathrm{V}]} & \text { Leitfähigkeit } \\ \varkappa & {[1 /(\Omega \mathrm{m})]} & \text { spezifischer Strömungswiderstand } \\ \varrho & {\left[\mathrm{kg} /\left(\mathrm{m}^{3} \mathrm{~s}\right)\right]} & \text { Vorzeichen der Festionenladung } \\ \omega & {[-]} & \text { Flächenanteil der L-Phase } \\ \zeta & {[-]} & \end{array}$

\section{Indices}

GG im Gleichgewicht

i Ionensorte $(1,2,3)$

ID Interdiffusion im Ionenaustauscher

L L-Phase

M Membran

S S-Phase

0 in der Ionenaustauscherpore

$\alpha \quad$ linke Membran

$\omega$ rechte Membran

\section{Literatur}

[1] Helfferich, F: Ionenaustauscher, Band I, VCH, Weinheim 1959.

[2] Korngold, E.: Desalination 16 (1975) S. 225/233.

[3] Strathmann, H.; Kock, K.: Effluent Free Electrolytic Regeneration of Ion Exchange Resins, Polymerseparation Media (Ed. Cooper, A. R.), Plenum Press, New York 1982.

[4] Deutsche Offenlegungsschrift DE 4016000 A 1, Anmeldetag: 18. 5. 1990 (Erfinder: J. Johann).

[5] Glabiszewski, F: Chemie-Technik (Heidelberg) 18 (1989) Nr. 1, S. 23/25.

[6] Turner, J. C. R.; Snowdon, C. B.: Chem. Eng. Sci. 23 (1968) S. 221/230.

[7] Loureiro, J. M.; Costa, C. A.; Rodrigues, A. E.: Chem. Eng. Sci. 43 (1988) S. 1115/1123.

[8] Gilliland, E. R.: Ind. Eng. Chem. 45 (1953) S. 330.

[9] Johann, J.: Dissertation, Universität Stuttgart 1992. 